\title{
Collisionless distribution function for the relativistic force-free Harris sheet
}

C. R. Stark and T. Neukirch

This is the author's final version of this article (c) AIP

Publishing. The final published version is available from doi: http://dx.doi.org/10.1063/1.3677268

Stark, C.R. and Neukirch, T. 2012. Collisionless distribution function for the relativistic force-free Harris sheet. Physics of Plasmas. 19(1): 012115. doi: 10.1063/1.3677268 


\section{Collisionless distribution function for the relativistic force-free Harris sheet}

C. R. Stark ${ }^{1, a)}$ and T. Neukirch ${ }^{1, b)}$

School of Mathematics and Statistics, University of St Andrews, St Andrews, KY16 9JT, United Kingdom

(Dated: 7 December 2011)

A self-consistent collisionless distribution function for the relativistic analogue of the force-free Harris sheet is presented. This distribution function is the relativistic generalization of the distribution function for the non-relativistic collisionless force-free Harris sheet recently found by Harrison and Neukirch [Phys. Rev. Lett. 102, 135003 (2009)] as it has the same dependence on the particle energy and canonical momenta. We present a detailed calculation which shows that the proposed distribution function generates the required current density profile (and thus magnetic field profile) in a frame of reference in which the electric potential vanishes identically. The connection between the parameters of the distribution function and the macroscopic parameters such as the current sheet thickness is discussed.

PACS numbers: 52.20.-j, 52.25.Xz, 52.55.-s, 52.65.Ff

Keywords: Suggested keywords

\footnotetext{
a)Electronic mail: craig@mcs.st-and.ac.uk

b)Electronic mail: thomas@mcs.st-and.ac.uk
} 


\section{INTRODUCTION}

Force-free plasma equilibria, i.e. plasma equilibria for which the current density is aligned with the magnetic field lines, are of great importance in both astrophysical and laboratory plasmas, in particular for modelling low- $\beta$ systems. Within the framework of magnetohydrodynamics (MHD) a large number of analytical force-free equilibria are known. ${ }^{1-3}$

For collisionless plasmas, with equilibria being solutions of the time-independent VlasovMaxwell (VM) equations, the situation is completely different. So far only a small number of one-dimensional (1D) collisionless force-free plasma equilibria is known, of which most belong to the class of linear force-free fields ${ }^{4-8}$. So far, analytical self-consistent distribution functions have been found only for one example of non-linear force-free fields, the force-free Harris sheet $^{9-12}$. Finding self-consistent force-free collisionless equilibria is difficult, because one is dealing with an inverse problem, i.e. find a solution of the Vlasov equation for a given magnetic field and electric current system (for a discussion of the problem see Ref. 10).

The equilibria mentioned in the previous paragraph have all been found for the nonrelativistic regime. It is the aim of this paper to investigate whether it is possible to generalize the distribution function found for the force-free Harris sheet into the relativistic regime. We define the relativistic force-free condition as $J^{\mu} F_{\mu \nu}=0$, where $J^{\mu}$ is the four-current density and $F_{\mu \nu}$ the electromagnetic four-tensor. If a frame of reference exists in which the electric field vanishes the relativistic force-free condition is identical with the non-relativistic force-free condition $\mathbf{J} \times \mathbf{B}=\mathbf{0}$ in this frame of reference.

Collisionless equilibria of the type we are trying to calculate in this paper could be of importance for investigations of physical processes like instabilities or magnetic reconnection in relativistic plasmas (see e.g. Refs 13-21). In order to find the relativistic generalization of the collisionless distribution function for the force-free Harris sheet, we shall use the relativistic version ${ }^{22-24}$ of the distribution function of the normal Harris $\operatorname{sheet}^{25}$ as a guide, together with the non-relativistic distribution function for the force-free Harris sheet. ${ }^{9,11}$

The paper is structured as follows: section II summarizes the mathematical framework of the non-relativistic force-free Harris sheet ${ }^{9}$. Section III then uses this as a basis for determining the distribution function for the relativistic force-free Harris sheet, and Section IV we present a discussion and our conclusions. 


\section{THE NON-RELATIVISTIC FORCE-FREE HARRIS SHEET}

In a 1D VM equilibria with translational symmetry, it is assumed that all plasma variables depend only on one spatial coordinate, here taken to be $z$ and that the magnetic flux density has components $B_{x}$ and $B_{y}$. The magnetic flux density components can be written in terms of the vector potential $\mathbf{A}=\left(A_{x}, A_{y}, A_{z}\right)$ where,

$$
\begin{aligned}
& B_{x}=-\frac{\mathrm{d} A_{y}}{\mathrm{~d} z}, \\
& B_{y}=\frac{\mathrm{d} A_{x}}{\mathrm{~d} z},
\end{aligned}
$$

and the electric field is the gradient of the electric potential $\phi$,

$$
\mathbf{E}=-\nabla \phi=-\frac{\mathrm{d} \phi}{\mathrm{d} z} \hat{\mathbf{z}}
$$

These relations automatically satisfy Faraday's law $\nabla \times \mathbf{E}=\mathbf{0}$ and Gauss' law for the magnetic flux density $\nabla \cdot \mathbf{B}=0$. Due to the symmetries of the system (time and spatial independence of $x$ and $y$ ) the three obvious constants of motion for each particle species are the Hamiltonian or particle energy for each species $s$,

$$
H_{s}=\frac{1}{2} m_{s}\left(v_{x}^{2}+v_{y}^{2}+v_{z}^{2}\right)+q_{s} \phi
$$

and the canonical momentum in the $x$ and $y$ directions respectfully,

$$
\begin{aligned}
& p_{x s}=m_{s} v_{x}+q_{s} A_{x}, \\
& p_{y s}=m_{s} v_{y}+q_{s} A_{y},
\end{aligned}
$$

where $m_{s}$ and $q_{s}$ are the mass and charge of each species. Here, we consider a plasma composed of two species of equal and opposite charge but of differing mass, that is, electrons and protons. All positive functions $f_{s}$ satisfying the appropriate conditions for the existence of the velocity moments and depending only on the constants of motion, $f_{s}=$ $f_{s}\left(H_{s}, p_{x s}, p_{y s}\right)$ solve the steady-state Vlasov equation. For a quasi-neutral plasma in force balance, Ampère's law can be written as

$$
\begin{aligned}
\frac{\mathrm{d}^{2} A_{x}}{\mathrm{~d} z^{2}} & =-\mu_{0} \frac{\partial P_{z z}}{\partial A_{x}}, \\
\frac{\mathrm{d}^{2} A_{y}}{\mathrm{~d} z^{2}} & =-\mu_{0} \frac{\partial P_{z z}}{\partial A_{y}},
\end{aligned}
$$


where $P_{z z}\left(A_{x}, A_{y}\right)$ is the $z z$-component of the plasma pressure tensor,

$$
P_{z z}=\sum_{s} \int_{-\infty}^{\infty} m_{s} v_{z}^{2} f_{s} \mathrm{~d}^{3} v
$$

The magnetic flux density components of the force-free Harris sheet are given by

$$
\begin{aligned}
& B_{x}=B_{0} \tanh (z / \lambda), \\
& B_{y}=\frac{B_{0}}{\cosh (z / \lambda)} .
\end{aligned}
$$

The $x$-component of the magnetic flux density has the same spatial structure as the Harris sheet, but whereas the Harris sheet is kept in force balance by pressure gradients, the forcefree Harris sheet maintains force balance via a magnetic shear $y$-component.

Assuming that the pressure takes the form $P_{z z}\left(A_{x}, A_{y}\right)=P_{1}\left(A_{x}\right)+P_{2}\left(A_{y}\right)$, equations (7) and (8) combined with the force-free condition $\left(B_{x}^{2}+B_{y}^{2}=\right.$ const.) gives the condition for force balance:

$$
\begin{aligned}
& \left(\frac{\mathrm{d} A_{x}}{\mathrm{~d} z}\right)^{2}+2 \mu_{0} P_{1}\left(A_{x}\right)=2 \mu_{0} P_{01}, \\
& \left(\frac{\mathrm{d} A_{y}}{\mathrm{~d} z}\right)^{2}+2 \mu_{0} P_{2}\left(A_{y}\right)=2 \mu_{0} P_{02},
\end{aligned}
$$

where $P_{01}$ and $P_{02}$ are constants. Solving these equations for $P_{1}\left(A_{x}\right)$ and $P_{2}\left(A_{y}\right)$ the plasma pressure for the force-free Harris sheet is

$$
P_{z z}=\frac{B_{0}^{2}}{2 \mu_{0}}\left[\frac{1}{2} \cos \left(\frac{2 A_{x}}{B_{0} \lambda}\right)+\exp \left(\frac{2 A_{y}}{B_{0} \lambda}\right)\right]+P_{03},
$$

where $P_{03}$ is a constant. Since the pressure is the sum of two independent functions that are a function of $A_{x}$ and $A_{y}$ respectively, the distribution function is assumed to be of the form

$$
f_{s}=\exp \left(-\beta_{s} H_{s}\right)\left[g_{1 s}\left(p_{x s}\right)+g_{2 s}\left(p_{y s}\right)\right]
$$

where the reciprocal thermal energy of species $s$ is $\beta_{s}=\left(k_{B} T_{s}\right)^{-1}$. Applying Channell's fourier transform method ${ }^{5}$, echoed in Harrison and Neukirch ${ }^{9}$, the plasma pressure integral can be solved for the distribution function $f_{s}$. Therefore, a collisionless distribution function for the force-free Harris sheet is

$$
\begin{aligned}
f_{s}= & \frac{n_{0, s}}{v_{t h, s}^{3}} \exp \left(-\beta_{s} H_{s}\right)\left[a_{s} \cos \left(\beta_{s} u_{x s} p_{x s}\right)\right. \\
& \left.+\exp \left(\beta_{s} u_{y s} p_{y s}\right)+b_{s}\right]
\end{aligned}
$$

where $v_{t h, s}=\left(m_{s} \beta_{s}\right)^{-1 / 2}$ is the thermal speed of species $s$ and $u_{x s}, u_{y s}, a_{s}$ and $b_{s}$ are constants. 


\section{RELATIVISTIC FORCE-FREE HARRIS SHEET}

If the thermal energy of the plasma, $k_{B} T$, approaches or exceeds the rest energy, $m c^{2}$, a non-relativistic treatment is no longer sufficient to describe the system. In a relativistic framework, $P^{\nu}$ are the components of the canonical momentum four-vector $\mathbf{P}=\mathbf{p}+q_{s} \mathbf{A}$, where: $\mathbf{p}=m \mathbf{w}=\left(E / c, p^{1}, p^{2}, p^{3}\right)$ is the four-momentum; $\mathbf{w}=\left(w^{0}, w^{1}, w^{2}, w^{3}\right)$ is the fourvelocity; and $\mathbf{A}=\left(\phi / c, A^{1}, A^{2}, A^{3}\right)$ is the four-potential. The Hamiltonian or particle energy for each species $s, H_{s}$ corresponds to the speed of light times the zeroth component of the canonical momentum four-vector $P^{0} c=E+q_{s} \phi$. The subsequent canonical components are now functions of the four-velocity, $P^{i}=m_{s} w^{i}+q_{s} A^{i}$. A relativistic analogue of the force-free Harris sheet distribution function can be written as

$$
\begin{aligned}
f_{s}= & f_{s 0} \exp \left(-\beta_{s} P^{0} c\right)\left[a_{s} \cos \left(\beta_{s} u_{x s} P^{1}\right)\right. \\
& \left.+\exp \left(\beta_{s} u_{y s} P^{2}\right)+b_{s}\right]
\end{aligned}
$$

where $f_{s 0}=n_{0 s} m_{s} \beta_{s} /(4 \pi c)$ and $n_{0 s}$ is the mean particle density. Details of the normalisation calculation are given in appendix A. Using the relation $\cos x=\frac{1}{2}\left(e^{i x}+e^{-i x}\right)$ we can recast the distribution function as

$$
\begin{aligned}
f_{s} & =f_{1 s}\left(w^{0}, w^{2}\right)+f_{2 s}\left(w^{0}, w^{1}\right)+f_{3 s}\left(w^{0}, w^{1}\right)+f_{4 s}\left(w^{0}\right) \\
& =f_{s 0}\left[c_{1 s} \exp \left(-c \beta_{s} m_{s}\left(w^{0}-u_{y s} w^{2} / c\right)\right)\right. \\
& +c_{2 s} \exp \left(-c \beta_{s} m_{s}\left(w^{0}+i u_{x s} w^{1} / c\right)\right) \\
& +c_{3 s} \exp \left(-c \beta_{s} m_{s}\left(w^{0}-i u_{x s} w^{1} / c\right)\right) \\
& \left.+c_{4 s} \exp \left(-c \beta_{s} m_{s} w^{0}\right)\right]
\end{aligned}
$$

where

$$
\begin{aligned}
& c_{1 s}=\exp \left(-\beta_{s} q_{s}\left(\phi-u_{y s} A^{2}\right)\right), \\
& c_{2 s}=\frac{a_{s}}{2} \exp \left(-\beta_{s} q_{s}\left(\phi+i u_{x s} A^{1}\right)\right), \\
& c_{3 s}=\frac{a_{s}}{2} \exp \left(-\beta_{s} q_{s}\left(\phi-i u_{x s} A^{1}\right)\right), \\
& c_{4 s}=b_{s} \exp \left(-\beta_{s} q_{s} \phi\right) .
\end{aligned}
$$

In the non-relativistic scenario the $z z$-component of the plasma pressure tensor is the key plasma parameter characterising the system; in the relativistic case it is the energymomentum tensor that is key. The energy-momentum tensor has components $T^{\alpha \beta}=$ 
$\left(T^{\alpha \beta}\right)_{\text {plasma }}+\left(T^{\alpha \beta}\right)_{e m}$, incorporating contributions to the energy and momentum from the kinetic and electromagnetic behaviour of the plasma. In this context, a relativistic force-free system corresponds to $T_{, \beta}^{\alpha \beta}=-J^{\beta} F_{\alpha \beta}=0$, where $F_{\alpha \beta}$ are the components of the Faraday tensor and $\mathbf{J}=\left(c \rho, J^{1}, J^{2}, J^{3}\right)$ is the four-current. In a frame where $\phi=0, \mathbf{B}=\left(B^{1}, B^{2}, 0\right)$ and $\mathbf{J}=\left(c \rho, J^{1}, J^{2}, 0\right)$, the only non-zero component of the Lorentz force is $J_{1} B^{2}-J_{2} B^{1}=0$.

Neglecting viscosity and heat conduction the 33-component of $\left(T^{\alpha \beta}\right)_{\text {plasma }}$, which we will refer to as the plasma pressure $P$, is given by

$$
\begin{aligned}
P & =c \sum_{s} m_{s} \int \mathrm{d}^{4} w\left(w^{3}\right)^{2} f_{s}(\mathbf{w}) \delta\left(w_{\nu} w^{\nu}-c^{2}\right) \\
& =c \sum_{s}\left(P_{1 s}+P_{2 s}+P_{3 s}+P_{4 s}\right) .
\end{aligned}
$$

Note that $\left(T^{\alpha \beta}\right)_{\text {plasma }}=p^{\alpha} N^{\beta}$, where $\mathbf{N}=n \mathbf{u}$ is the number flux four-vector and $n$ is the number density. To solve the first pressure integral $P_{1 s}$ we will perform a coordinate transformation defined by the transformation matrix

$$
\left[\Lambda_{\alpha}^{\bar{\beta}}\right]=\left[\begin{array}{cccc}
\gamma_{1 s} & 0 & -u_{y s} \gamma_{1 s} / c & 0 \\
0 & 1 & 0 & 0 \\
-u_{y s} \gamma_{1 s} / c & 0 & \gamma_{1 s} & 0 \\
0 & 0 & 0 & 1
\end{array}\right]
$$

where $\gamma_{1 s}=\left(1-u_{y s}^{2} / c^{2}\right)^{-1 / 2}, w^{\bar{\beta}}=\Lambda_{\alpha}^{\bar{\beta}} w^{\alpha}$ and $w^{\alpha}=\Lambda_{\bar{\beta}}^{\alpha} w^{\bar{\beta}}$ such that $\Lambda_{\alpha}^{\bar{\beta}} \Lambda_{\bar{\beta}}^{\alpha}=1$. Note that this coordinate transformation, and following transformations, are used as a means to evaluate the integral and do not physically correspond to a Lorentz boost. The Jacobian of the system is simply $J=1$ therefore, $\mathrm{d} w^{\bar{\beta}}=\mathrm{d} w^{\alpha}$ and $f_{1 s}\left(w^{0}, w^{2}\right)=f_{1 s}\left(w^{\overline{0}}\right)$. Making the change of variables yields

$$
P_{1 s}=m_{s} \int \mathrm{d}^{4} \bar{w}\left(w^{\overline{3}}\right)^{2} f_{1 s}\left(w^{\overline{0}}\right) \delta\left(w_{\nu} w^{\nu}-c^{2}\right)
$$

which can be written as

$$
P_{1 s}=m_{s} \int \mathrm{d}^{3} \bar{w} \frac{\left(w^{\overline{3}}\right)^{2}}{w^{\overline{0}}} f_{1 s}\left(w^{\overline{0}}\right)
$$

where

$$
f_{1 s}=f_{s 0} c_{1 s} \exp \left(-c \beta_{s} m_{s} w^{\overline{0}} / \gamma_{1 s}\right)
$$

A convenient way of expressing $w^{\overline{0}}$ can be obtained using the inner product of the fourvelocity with itself, $\mathbf{w} \cdot \mathbf{w}=g_{\mu \nu} w^{\nu} w_{\mu}$ which gives $w^{\overline{0}}=\sqrt{c^{2}+(w)^{2}}$. Note the metric used 
here is defined as $g_{\mu \nu}=\operatorname{diag}(+1,-1,-1,-1)$. The integral can now be easily evaluated by changing to spherical coordinates, making use of the Jüttner transformation $(w / c=\sinh x)$ and using the known integral ${ }^{26}$

$$
\begin{gathered}
\int_{0}^{\infty} \sinh ^{2 \nu} x \exp (-\beta \cosh x) \mathrm{d} x \\
=\frac{1}{\sqrt{\pi}}\left(\frac{2}{\beta}\right)^{\nu} \Gamma\left(\nu+\frac{1}{2}\right) K_{\nu}(\beta),
\end{gathered}
$$

where $K$ is the modified Bessel Function of the second kind. Therefore

$$
P_{1 s}=\frac{k_{1 s} n_{0 s}}{\beta_{s} c} \exp \left(-\beta_{s} q_{s}\left(\phi-u_{y s} A^{2}\right)\right)
$$

where $k_{1 s}=\gamma_{1 s}^{2} K_{2}\left(\Lambda_{1 s}\right)$ and $\Lambda_{i s}=m_{s} c^{2} \beta_{s} / \gamma_{i s}$. Similarly $P_{2 s}$ and $P_{3 s}$ can be evaluated using the following coordinate transformation

$$
\left[\Delta_{\alpha}^{\bar{\beta}}\right]=\left[\begin{array}{cccc}
\gamma_{2 s} & \pm i u_{x s} \gamma_{2 s} / c & 0 & 0 \\
\pm i u_{x s} \gamma_{2 s} / c & \gamma_{2 s} & 0 & 0 \\
0 & 0 & 1 & 0 \\
0 & 0 & 0 & 1
\end{array}\right]
$$

where $\gamma_{2 s}=\left(1+u_{x s}^{2} / c^{2}\right)^{-1 / 2}, w^{\bar{\beta}}=\Delta_{\alpha}^{\bar{\beta}} w^{\alpha}$ and $w^{\alpha}=\Delta_{\bar{\beta}}^{\alpha} w^{\bar{\beta}}$ such that $\Delta_{\alpha}^{\bar{\beta}} \Delta_{\bar{\beta}}^{\alpha}=1$. The Jacobian is again $J=1$, with $f_{2 s}\left(w^{0}, w^{1}\right)=f_{2 s}\left(w^{\overline{0}}\right)$ and $f_{3 s}\left(w^{0}, w^{1}\right)=f_{3 s}\left(w^{\overline{0}}\right)$. This yields

$$
\begin{aligned}
& P_{2 s}=\frac{k_{2 s} n_{0 s}}{2 \beta_{s} c} \exp \left(-\beta_{s} q_{s}\left(\phi+i u_{x s} A^{1}\right)\right), \\
& P_{3 s}=\frac{k_{2 s} n_{0 s}}{2 \beta_{s} c} \exp \left(-\beta_{s} q_{s}\left(\phi-i u_{x s} A^{1}\right)\right),
\end{aligned}
$$

where $k_{2 s}=a_{s} \gamma_{2 s}^{2} K_{2}\left(\Lambda_{2 s}\right)$. The final pressure integral $P_{4 s}$ can be trivially evaluated, without any need for a coordinate transformation, using Eq. (29) yielding

$$
P_{4 s}=\frac{k_{3 s} n_{0 s}}{\beta_{s} c} \exp \left(-\beta_{s} q_{s} \phi\right)
$$

where $k_{3 s}=b_{s} K_{2}\left(\Lambda_{3 s}\right)$ and $\Lambda_{3 s}=m_{s} c^{2} \beta_{s}$. The total plasma pressure is then

$$
\begin{aligned}
P & =\sum_{s} \frac{n_{0 s} k_{1 s}}{\beta_{s}} \exp \left(-\beta_{s} q_{s} \phi\right)\left[\exp \left(\beta_{s} q_{s} u_{y s} A^{2}\right)\right. \\
& \left.+\left(k_{2 s} / k_{1 s}\right) \cos \left(\beta_{s} q_{s} u_{x s} A^{1}\right)+k_{3 s} / k_{1 s}\right] .
\end{aligned}
$$

The charge density $\rho$ is given by (see Eq.(B5)),

$$
\rho=-\frac{\partial P}{\partial \phi}=\sum_{s} q_{s} \exp \left(-\beta_{s} q_{s} \phi\right) N_{s}\left(A^{1}, A^{2}\right),
$$


Relativistic force-free Harris sheet

where,

$$
\begin{aligned}
N_{s}\left(A^{1}, A^{2}\right)= & n_{0 s} k_{1 s}\left[\exp \left(\beta_{s} q_{s} u_{y s} A^{2}\right)\right. \\
& +\left(k_{2 s} / k_{1 s}\right) \cos \left(\beta_{s} q_{s} u_{x s} A^{1}\right) \\
& \left.+k_{3 s} / k_{1 s}\right] .
\end{aligned}
$$

A charge neutral plasma requires that $\rho=0$, hence

$$
\phi=\frac{1}{e\left(\beta_{i}+\beta_{e}\right)} \ln \left(\frac{N_{i}}{N_{e}}\right) .
$$

The condition of vanishing electric field is satisfied by $N_{e}=N_{i}$, which is true if

$$
\begin{aligned}
\beta_{i} u_{y i} & =-\beta_{e} u_{y e}, \\
\beta_{i}\left|u_{x i}\right| & =\beta_{e}\left|u_{x e}\right|, \\
n_{0 i} k_{1 i} & =n_{0 e} k_{1 e}=n_{0}, \\
k_{2 i} / k_{1 i} & =k_{2 e} / k_{1 e}=a, \\
k_{3 i} / k_{1 i} & =k_{3 e} / k_{1 e}=b .
\end{aligned}
$$

As a result the plasma pressure is given by

$$
\begin{aligned}
P= & \frac{\left(\beta_{i}+\beta_{e}\right)}{\beta_{i} \beta_{e}} n_{0}\left[\exp \left(-\beta_{e} e u_{y e} A^{2}\right)\right. \\
& \left.+a \cos \left(\beta_{e} e u_{x e} A^{1}\right)+b\right] .
\end{aligned}
$$

The $x$ - and $y$ - components of the current density are calculated using Eqs. (B6) and (B7) (see Appendix B)

$$
\begin{aligned}
& J^{1}=\frac{\partial P}{\partial A^{1}}=-\frac{\left(\beta_{i}+\beta_{e}\right)}{\beta_{i}} n_{0} a e u_{x e} \sin \left(\beta_{e} e u_{x e} A^{1}\right) \\
& J^{2}=\frac{\partial P}{\partial A^{2}}=-\frac{\left(\beta_{i}+\beta_{e}\right)}{\beta_{i}} n_{0} e u_{y e} \exp \left(-\beta_{e} e u_{y e} A^{2}\right) .
\end{aligned}
$$

The corresponding, self-consistent vector potential can be obtained via Ampère's Law,

$$
\begin{aligned}
& \frac{\mathrm{d}^{2} A^{1}}{\mathrm{~d} z^{2}}=-\mu_{0} J^{1}, \\
& \frac{\mathrm{d}^{2} A^{2}}{\mathrm{~d} z^{2}}=-\mu_{0} J^{2} .
\end{aligned}
$$

The resulting vector potential components can be written as,

$$
\begin{aligned}
& A^{1}=\alpha_{1} \arctan \left(\exp \left(z / \lambda_{1}\right)\right), \\
& A^{2}=\alpha_{2} \ln \left(\cosh ^{2}\left(z / \lambda_{2}\right)\right),
\end{aligned}
$$


Relativistic force-free Harris sheet

and the magnetic flux density components are

$$
\begin{aligned}
B^{1} & =-\left(2 \alpha_{2} / \lambda_{2}\right) \tanh \left(z / \lambda_{2}\right), \\
B^{2} & =\frac{\alpha_{1} /\left(2 \lambda_{1}\right)}{\cosh \left(z / \lambda_{1}\right)}
\end{aligned}
$$

where

$$
\begin{aligned}
& \alpha_{1}=4 /\left(\beta_{e} e u_{x e}\right), \\
& \alpha_{2}=1 /\left(\beta_{e} e u_{y e}\right), \\
& \lambda_{1}=\left(\frac{\beta_{i}}{\beta_{e}\left(\beta_{i}+\beta_{e}\right) a \mu_{0} n_{0} e^{2} u_{x e}^{2}}\right)^{1 / 2}, \\
& \lambda_{2}=\left(\frac{2 \beta_{i}}{\beta_{e}\left(\beta_{i}+\beta_{e}\right) \mu_{0} n_{0} e^{2} u_{y e}^{2}}\right)^{1 / 2} .
\end{aligned}
$$

For a force-free system we require $J_{1} B^{2}-J_{2} B^{1}=0$. This condition is satisfied provided $4 \alpha_{2}= \pm \alpha_{1}\left(\alpha=\left|\alpha_{1}\right|\right)$ and $\lambda_{1}=\lambda_{2}=\lambda$ which implies $u_{x e}=-u_{y e}$ and $a=1 / 2$. Therefore, the relativistic force-free Harris sheet is

$$
\begin{aligned}
& B^{1}=B_{0} \tanh (z / \lambda), \\
& B^{2}=\frac{B_{0}}{\cosh (z / \lambda)},
\end{aligned}
$$

where $B_{0}=\alpha /(2 \lambda)$. The relationship between the microscopic and macroscopic parameters of the equilibria can be deduced by comparing Eq. (14) to Eq. (44). This yields

$$
\begin{aligned}
\frac{B_{0}^{2}}{2 \mu_{0}} & =n_{0} \frac{\left(\beta_{i}+\beta_{e}\right)}{\beta_{e} \beta_{i}}, \\
a & =\frac{1}{2}, \\
P_{03} & =n_{0} \frac{\left(\beta_{i}+\beta_{e}\right)}{\beta_{e} \beta_{i}} b, \\
\frac{2}{\left|B_{0}\right| \lambda} & =e \beta_{e}\left|u_{x e}\right|=e \beta_{i}\left|u_{x i}\right|, \\
\frac{2}{B_{0} \lambda} & =-e \beta_{e} u_{y e}=e \beta_{i} u_{y i},
\end{aligned}
$$

where we have assumed that $\lambda$ is positive and $B_{0}$ can be negative. Following Neukirch et $a l .{ }^{11}$ the connection with the original Harris sheet can be made using Eq. (59) and Eq. (63) to obtain an expression for $\lambda$,

$$
\lambda=\left(\frac{2\left(\beta_{e}+\beta_{i}\right)}{\mu_{0} e^{2} \beta_{e} \beta_{i} n_{0}\left(u_{y i}-u_{y e}\right)^{2}}\right)^{1 / 2}
$$


Therefore, the relativistic force-free Harris sheet is equivalent to the non-relativistic forcefree Harris sheet ${ }^{9-12}$ (and consistent with the relativistic Harris sheet ${ }^{22}$ ) where we can now formally access the relativistic regime, $m_{s} c^{2} \beta_{s} \ll 1$.

When $m_{s} c^{2} \beta_{s} \gg 1$ the relativistic solution reduces to its non-relativistic counterpart where,

$$
\begin{aligned}
n_{0} & =n_{0 s} \gamma_{1 s}^{2} K_{2}\left(\Lambda_{1 s}\right) \approx \alpha_{1} \exp \left(\frac{m_{s} \beta_{s} u_{y s}^{2}}{2}\right) \\
a & =\frac{a_{s} \gamma_{2 s}^{2} K_{2}\left(\Lambda_{2 s}\right)}{\gamma_{1 s}^{2} K_{2}\left(\Lambda_{1 s}\right)} \\
& \approx \alpha_{2} \exp \left(-\frac{m_{s} \beta_{s}}{2}\left(u_{x s}^{2}+u_{y s}^{2}\right)\right) \\
b & =\frac{b_{s} K_{2}\left(\Lambda_{3 s}\right)}{\gamma_{1 s}^{2} K_{2}\left(\Lambda_{1 s}\right)} \approx \alpha_{3} \exp \left(-\frac{m_{s} \beta_{s} u_{y s}^{2}}{2}\right)
\end{aligned}
$$

and

$$
\begin{aligned}
\alpha_{1}= & n_{0 s}\left(1+u_{y s}^{2} / c^{2}\right)\left(1+u_{y s}^{2} /\left(4 c^{2}\right)\right) \\
& \times\left(\frac{\pi}{2 m_{s} \beta_{s} c^{2}}\right)^{1 / 2} \exp \left(-m_{s} \beta_{s} c^{2}\right), \\
\alpha_{2}= & a_{s} \frac{\left(1-u_{x s}^{2} / c^{2}\right)}{\left(1+u_{y s}^{2} / c^{2}\right)} \frac{\left(1-u_{x s}^{2} /\left(4 c^{2}\right)\right)}{\left(1+u_{y s}^{2} /\left(4 c^{2}\right)\right)} \\
\alpha_{3}= & b_{s} \frac{\left(1-u_{y s}^{2} /\left(4 c^{2}\right)\right)}{\left(1+u_{y s}^{2} / c^{2}\right)}
\end{aligned}
$$

This is consistent with Neukirch et al. ${ }^{11}$. In the ultra-relativistic regime $m_{s} c^{2} \beta_{s} \ll 1$ and

$$
\begin{aligned}
n_{0} & \approx \frac{2 n_{0 s}}{\left(m_{s} c^{2} \beta_{s}\right)^{2}}\left(1-u_{y s}^{2} / c^{2}\right)^{-2}, \\
a & \approx a_{s}\left(\frac{1-u_{y s}^{2} / c^{2}}{1+u_{x s}^{2} / c^{2}}\right)^{2}=\frac{1}{2} \\
b & \approx b_{s}\left(1-u_{y s}^{2} / c^{2}\right)^{2} .
\end{aligned}
$$

The general solution is constrained by the condition

$$
a=\frac{a_{s} \gamma_{2 s}^{2} K_{2}\left(\Lambda_{2 s}\right)}{\gamma_{1 s}^{2} K_{2}\left(\Lambda_{1 s}\right)}=\frac{1}{2}
$$

where $a_{s}$ is a free parameter. This condition dictates the allowable values of $u_{x s}=-u_{y s}=u_{s}$ for a given $m_{s} c^{2} \beta_{s}$, Fig. 1 exhibits the relationship between $u_{s} / c$ and $m_{s} c^{2} \beta_{s}$ for $a_{s}=1$, giving a hint of the general dependence for arbitrary $a_{s}$. 


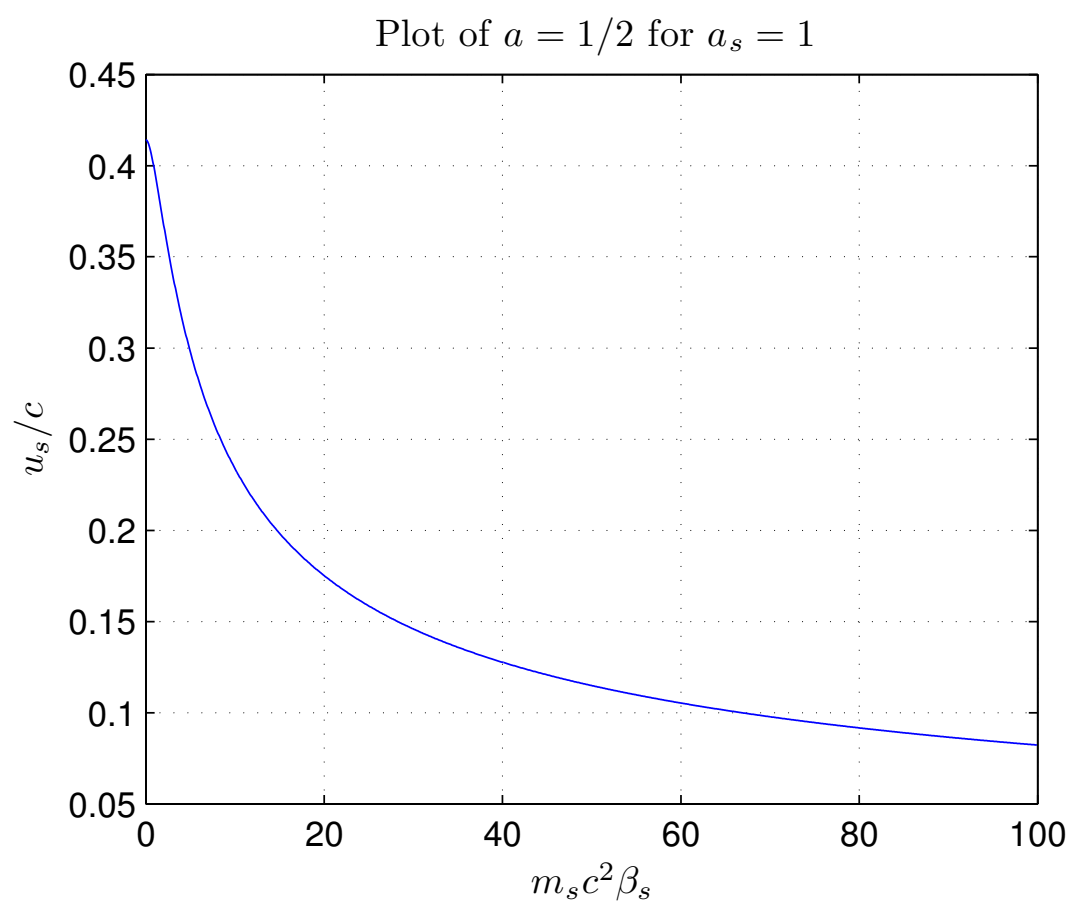

FIG. 1. Plot of $u_{s} / c$ against $m_{s} c^{2} \beta_{s}$ for $a_{s}=1$, exhibiting the relationship $a=1 / 2$ that constrains the relativistic force-free Harris sheet.

\section{SUMMARY AND CONCLUSIONS}

Recently the first nonlinear force-free, non-relativistic VM equilibrium for the force-free Harris sheet was reported by Harrison and Neukirch ${ }^{9}$. If the thermal energy of the plasma approaches or exceeds its rest energy, a non-relativistic treatment is no longer sufficient and a relativistic analogue must be sought. This paper has presented a collisionless distribution function for the relativistic force-free Harris sheet. Mirroring the non-relativistic solution ${ }^{9-11}$, where the properties of the pressure tensor were exploited, the energy-momentum tensor $T^{\alpha \beta}$ was wielded in an equivalent role ${ }^{27}$, allowing the calculation of the equilibrium. In our calculation, we restrict ourselves to a frame where the electric potential vanishes $(\phi=0)$, $\mathbf{B}=\left(B^{1}, B^{2}, 0\right)$, and $\mathbf{J}=\left(c \rho, J^{1}, J^{2}, 0\right)$, where the only non-zero component of the Lorentz force is $J_{1} B^{2}-J_{2} B^{1}=0$. The condition of vanishing electric field is true only in one frame of reference; in a moving frame $\phi$ is non-zero. The resulting relativistic force-free Harris sheet is identical to its non-relativistic counterpart but now the relativistic regime where $m_{s} c^{2} \beta_{s} \ll 1$, can be formally accessed.

Alternative methods of studying relativistic VM equilibria have also been developed ${ }^{28-34}$. 
Suzuki explored possible equilibria by describing the deviation of the distribution function from a Maxwell-Jüttner distribution using orthogonal polynomial series ${ }^{28}$. Using this novel method a new, two dimensional equilibrium was reported. Kocharovsky and co-workers use the method of invariants of particle motion to find exact solutions of the VM system for arbitrary particle energy distributions ${ }^{29-31}$. There technique allows for the description of multicomponent plasmas, that may be relativistic or not, for a general magnetic geometry ${ }^{29}$.

Relativistic equilibria have also been studied extensively within the context of plasma pinches and electron beams used in fusion and laboratory plasmas ${ }^{35-42}$. In these investigations an electron beam, described by a prescribed distribution function, is embedded in a background plasma permeated by a background magnetic field. The resulting electromagnetic fields are self-consistently calculated yielding stable Vlasov equilibria. A variety of tailored seed distribution functions have been investigated such as monoenergetic distributions ${ }^{35,36}$; warm beams (i.e. a drifting Maxwellian) ${ }^{37,38}$; and helical (force-free) beams (i.e. a combination of axial and azimuthal configurations) ${ }^{38-42}$.

The work presented here lays the critical bedrock for further investigations of plasma phenomena such as waves, instabilities and magnetic reconnection. In particular the distribution function reported here can be used as the initial conditions for numerical investigations of magnetic reconnection in relativistic, collisionless plasmas.

\section{ACKNOWLEDGMENTS}

The Authors gratefully acknowledge funding from the Leverhulme Trust (F/00268/BB). The authors would like to thank the anonymous referee whose valuable feedback helped to improve this manuscript. 


\section{Appendix A: Normalisation Constant, $f_{s 0}$}

Consider the distribution function,

$$
\begin{aligned}
f_{s} & =f_{1 s}\left(w^{0}, w^{2}\right)+f_{2 s}\left(w^{0}, w^{1}\right)+f_{3 s}\left(w^{0}, w^{1}\right)+f_{4 s}\left(w^{0}\right) \\
& =f_{s 0}\left[c_{1 s} \exp \left(-c \beta_{s} m_{s}\left(w^{0}-u_{y s} w^{2} / c\right)\right)\right. \\
& +c_{2 s} \exp \left(-c \beta_{s} m_{s}\left(w^{0}+i u_{x s} w^{1} / c\right)\right) \\
& +c_{3 s} \exp \left(-c \beta_{s} m_{s}\left(w^{0}-i u_{x s} w^{1} / c\right)\right) \\
& \left.+c_{4 s} \exp \left(-c \beta_{s} m_{s} w^{0}\right)\right]
\end{aligned}
$$

where

$$
\begin{aligned}
c_{1 s} & =\exp \left(-\beta_{s} q_{s}\left(\phi-u_{y s} A^{2}\right)\right), \\
c_{2 s} & =\frac{a_{s}}{2} \exp \left(-\beta_{s} q_{s}\left(\phi+i u_{x s} A^{1}\right)\right), \\
c_{3 s} & =\frac{a_{s}}{2} \exp \left(-\beta_{s} q_{s}\left(\phi-i u_{x s} A^{1}\right)\right), \\
c_{4 s} & =b_{s} \exp \left(-\beta_{s} q_{s} \phi\right) .
\end{aligned}
$$

The four-current density $J^{\mu}=\left(c \rho, J^{1}, J^{2}, J^{3}\right)$ is defined as

$$
J^{\mu}=c \sum_{s} q_{s} \int \mathrm{d}^{4} w w^{\mu} f_{s} \delta\left(w_{\nu} w^{\nu}-c^{2}\right) .
$$

To evaluate the normalisation constant $f_{s 0}$, we make use of the zeroth component of the four-current (the charge density $\rho=\sum_{s} q_{s} n_{s}$ ) to calculate the charge number density,

$$
\begin{aligned}
n_{0 s} \tilde{n} & =\int \mathrm{d}^{4} w w^{0} f_{s} \delta\left(w_{\nu} w^{\nu}-c^{2}\right) \\
& =n_{1 s}+n_{2 s}+n_{3 s}+n_{4 s},
\end{aligned}
$$

where $n_{0 s}$ is the mean particle density. The first charge number density integral can be written as

$$
\begin{aligned}
n_{1 s} & =\int \mathrm{d}^{4} w w^{0} f_{1 s}\left(w^{0}, w^{2}\right) \delta\left(w_{\nu} w^{\nu}-c^{2}\right) \\
& =\int \mathrm{d}^{3} w f_{1 s}\left(w^{0}, w^{2}\right) .
\end{aligned}
$$

To solve the first density integral a coordinate transformation defined by Eq.(25) is performed,

$$
n_{1 s}=\gamma_{1 s} \int \mathrm{d}^{3} \bar{w} f_{1 s}\left(w^{\overline{0}}\right)
$$


Recall that the new set of variables are denoted by an overbar, where

$$
f_{1 s}=f_{s 0} c_{1 s} \exp \left(-c \beta_{s} m_{s} w^{\overline{0}} / \gamma_{1}\right)
$$

As detailed in section III, the inner product of the four-velocity with itself can be used to obtain $w^{\overline{0}}=\sqrt{c^{2}+(w)^{2}}$. The integral can now be simply evaluated after changing to spherical coordinates, making use of the Jüttner transformation, $w / c=\sinh x$, and using the known definite integral ${ }^{26}$

$$
\int_{0}^{\infty} \exp (-\beta \cosh x) \sinh \gamma x \sinh x \mathrm{~d} x=\frac{\gamma}{\beta} K_{\gamma}(\beta),
$$

where $K$ is the modified Bessel function of the second kind. Therefore the first integral becomes

$$
n_{1 s}=\frac{4 \pi c^{3} f_{s 0} \gamma_{1 s} K_{2}\left(\Lambda_{1 s}\right)}{\Lambda_{1 s}} \exp \left(-\beta_{s} q_{s}\left(\phi-u_{y s} A^{2}\right)\right) .
$$

The remaining density integrals can be evaluated in a similar fashion using Eq. (A12). Whereas $n_{2 s}$ and $n_{3 s}$ make us of the coordinate transformation defined by Eq. (31), this is not required for evaluating $n_{4 s}$. Therefore,

$$
\begin{aligned}
\frac{n_{0 s} \tilde{n}}{f_{s 0}}= & \frac{4 \pi c}{m_{s} \beta_{s}} \exp \left(-\beta_{s} q_{s} \phi\right)\left[\gamma_{1 s}^{2} K_{2}\left(\Lambda_{1 s}\right) \exp \left(\beta_{s} q_{s} u_{y s} A^{2}\right)\right. \\
& +a_{s} \gamma_{2 s}^{2} K_{2}\left(\Lambda_{2 s}\right) \cos \left(\beta_{s} q_{s} u_{x s} A^{1}\right) \\
& \left.+b_{s} K_{2}\left(\Lambda_{3 s}\right)\right] .
\end{aligned}
$$

By letting

$$
\begin{aligned}
\tilde{n}= & \exp \left(-\beta_{s} q_{s} \phi\right)\left[\gamma_{1 s}^{2} K_{2}\left(\Lambda_{1 s}\right) \exp \left(\beta_{s} q_{s} u_{y s} A^{2}\right)\right. \\
& +a_{s} \gamma_{2 s}^{2} K_{2}\left(\Lambda_{2 s}\right) \cos \left(\beta_{s} q_{s} u_{x s} A^{1}\right) \\
& \left.+b_{s} K_{2}\left(\Lambda_{3 s}\right)\right]
\end{aligned}
$$

we find that the normalisation constant is given by

$$
f_{s 0}=\frac{n_{0 s} m_{s} \beta_{s}}{4 \pi c}
$$

\section{Appendix B: $J^{\gamma}=\partial P / \partial A^{\gamma}$ Relations}

This appendix details the derivation of the $J^{\gamma}=\partial P / \partial A^{\gamma}$ relations required for the calculation of the relativistic force-free Harris sheet following the work of $\mathrm{Otto}^{27}$. In the 
kinetic treatment outlined in this manuscript the $\delta \delta$-component of the energy-momentum tensor $\left(\left(T^{\alpha \beta}\right)_{\text {plasma }}=p^{\alpha} N^{\beta}\right)$ is defined by the integral

$$
P=m c \int \mathrm{d}^{4} w\left(w^{\delta}\right)^{2} f \delta\left(w_{\nu} w^{\nu}-c^{2}\right) .
$$

This is the plasma pressure. Note that for clarity we suspend momentarily the use of the particle species subscript $s$. For a distribution function $f=f\left(P^{\nu}\right)$, we make the substitution $h=2 w^{\delta}$ (hence $\mathrm{d} w^{\delta}=\frac{1}{2} \mathrm{~d} h$ ) in the subsequent calculations. Taking the derivative of the plasma pressure with respect to $A^{\gamma}$ yields

$$
\begin{aligned}
\frac{\partial P}{\partial A^{\gamma}} & =m c \int \mathrm{d} w^{\alpha} \mathrm{d} w^{\beta} \mathrm{d} w^{\gamma} \mathrm{d} w^{\delta}\left(w^{\delta}\right)^{2} \frac{\partial f}{\partial A^{\gamma}} \delta\left(w_{\nu} w^{\nu}-c^{2}\right) \\
& =\frac{m c}{8} \int \mathrm{d} w^{\alpha} \mathrm{d} w^{\beta} \mathrm{d} w^{\gamma} \mathrm{d} h h^{2} \frac{\partial P^{\gamma}}{\partial A^{\gamma}} \frac{\partial f}{\partial P^{\gamma}} \delta(g(\mathbf{w})) \\
& =\frac{q c}{8} \int \mathrm{d} w^{\alpha} \mathrm{d} w^{\beta} \mathrm{d} w^{\gamma} \mathrm{d} h h^{2} \frac{\partial f}{\partial w^{\gamma}} \delta(g(\mathbf{w})) \\
& =\frac{q c}{2} \int \mathrm{d} w^{\alpha} \mathrm{d} w^{\beta} \mathrm{d} w^{\gamma}\left(g(\mathbf{w})+h^{2} / 4\right) \frac{\partial f}{\partial w^{\gamma}}
\end{aligned}
$$

where $g(\mathbf{w})$ is defined as

$$
g(\mathbf{w})=\left(w^{\alpha}\right)^{2}-\left(w^{\beta}\right)^{2}-\left(w^{\gamma}\right)^{2}-\frac{h^{2}}{4}-c^{2} .
$$

This can be simplified by integrating the $w^{\gamma}$ integral by parts,

$$
\begin{aligned}
\frac{\partial P}{\partial A^{\gamma}}= & \frac{q c}{2}\left(\left[\left(w_{\nu} w^{\nu}+\left(w^{\delta}\right)^{2}-c^{2}\right) f\right]_{-\infty}^{+\infty} \pm 2 \int w^{\gamma} f \mathrm{~d} w^{\gamma}\right) \\
& \times \int \mathrm{d} w^{\alpha} \mathrm{d} w^{\beta} \\
= & \pm q c \int \mathrm{d} w^{\alpha} \mathrm{d} w^{\beta} \mathrm{d} w^{\gamma} w^{\gamma} f \\
= & \pm q c \int \mathrm{d} w^{\delta} \delta\left(w_{\nu} w^{\nu}-c^{2}\right) \int \mathrm{d} w^{\alpha} \mathrm{d} w^{\beta} \mathrm{d} w^{\gamma} w^{\gamma} f \\
= & \pm q c \int \mathrm{d}^{4} w w^{\gamma} f \delta\left(w_{\nu} w^{\nu}-c^{2}\right) \\
= & \pm J^{\gamma} .
\end{aligned}
$$

Note that $\int \mathrm{d} w^{\delta} \delta\left(w_{\nu} w^{\nu}-c^{2}\right)=1$. Where the lower sign corresponds to the zeroth component case $(\gamma=0)$. Therefore

$$
\begin{aligned}
\rho & =-\frac{\partial P}{\partial \phi}, \\
J^{1} & =\frac{\partial P}{\partial A^{1}} \\
J^{2} & =\frac{\partial P}{\partial A^{2}} .
\end{aligned}
$$


Relativistic force-free Harris sheet

\section{REFERENCES}

${ }^{1}$ D. Biskamp, Nonlinear Magnetohydrodynamics, Cambridge monographs on plasma physics (Cambridge University Press, 1997).

${ }^{2}$ E. Priest, Solar magneto-hydrodynamics, Geophysics and astrophysics monographs (D. Reidel Pub. Co., 1984).

${ }^{3} \mathrm{G}$. Marsh, Force-free magnetic fields: solutions, topology and applications (World Scientific, 1996).

${ }^{4}$ A. Sestero, Physics of Fluids 10, 193 (1967).

${ }^{5}$ P. J. Channell, Physics of Fluids 19, 1541 (1976).

${ }^{6}$ N. A. Bobrova and S. I. Syrovatskii, Soviet Journal of Experimental and Theoretical Physics Letters 30, 535 (1979).

${ }^{7}$ D. Correa-Restrepo and D. Pfirsch, Phys. Rev. E 47, 545 (1993).

${ }^{8}$ N. A. Bobrova, S. V. Bulanov, J. I. Sakai, and D. Sugiyama, Physics of Plasmas 8, 759 (2001).

${ }^{9}$ M. G. Harrison and T. Neukirch, Physical Review Letters 102, 135003 (2009), arXiv:0812.1240 [physics.plasm-ph].

${ }^{10}$ M. G. Harrison and T. Neukirch, Physics of Plasmas 16, 022106 (2009), arXiv:0811.4604 [physics.plasm-ph].

${ }^{11}$ T. Neukirch, F. Wilson, and M. G. Harrison, Physics of Plasmas 16, 122102 (2009), arXiv:0911.0081 [physics.plasm-ph].

${ }^{12}$ F. Wilson and T. Neukirch, Physics of Plasmas 18, 082108 (2011), arXiv:1108.0534 [physics.plasm-ph].

${ }^{13}$ A. Otto and K. Schindler, Plasma Physics and Controlled Fusion 26, 1525 (1984).

${ }^{14}$ E. G. Blackman and G. B. Field, Physical Review Letters 72, 494 (1994).

${ }^{15}$ S. Zenitani and M. Hoshino, Astrophysical Journal Letters 562, L63 (2001).

${ }^{16}$ M. Lyutikov and D. Uzdensky, Astrophysical Journal 589, 893 (2003), arXiv:astro$\mathrm{ph} / 0210206$.

${ }^{17}$ C. H. Jaroschek, H. Lesch, and R. A. Treumann, Astrophysical Journal Letters 605, L9 (2004).

${ }^{18}$ N. Watanabe and T. Yokoyama, Astrophysical Journal Letters 647, L123 (2006), arXiv:astro-ph/0607285. 
${ }^{19}$ S. S. Komissarov, M. Barkov, and M. Lyutikov, Monthly Notices of the Royal Astronomical Society 374, 415 (2007), arXiv:astro-ph/0606375.

${ }^{20}$ S. Zenitani and M. Hoshino, Astrophysical Journal 670, 702 (2007), arXiv:0708.1000.

${ }^{21}$ S. Zenitani and M. Hesse, Astrophysical Journal 684, 1477 (2008), arXiv:0805.4286.

${ }^{22}$ F. C. Hoh, Physics of Fluids 9, 277 (1966).

${ }^{23}$ B. Buti, Physics of Fluids 5, 1 (1962).

${ }^{24}$ B. Buti, Physics of Fluids 6, 89 (1963).

${ }^{25}$ E. Harris, Il Nuovo Cimento (1955-1965) 23, 115 (1962), 10.1007/BF02733547.

${ }^{26}$ Gradshteŭ, Table of integrals, series, and products.

${ }^{27}$ A. Otto, Ein Energieprinzip für Stossfreie Relativistische Plasmen, Ph.D. thesis, Institut für Theoretische Physik IV der Ruhr-Universität Bochum (1983).

${ }^{28}$ A. Suzuki, Physics of Plasmas 15, 072107 (2008), arXiv:0806.0898 [physics.plasm-ph].

${ }^{29}$ V. V. Kocharovsky, V. V. Kocharovsky, and V. J. Martyanov, Physical Review Letters 104, 215002 (2010).

${ }^{30}$ V. V. Kocharovsky, V. V. Kocharovsky, and V. Y. Martyanov, Radiophysics and Quantum Electronics 52, 79 (2009).

${ }^{31}$ V. Y. Mart'yanov, V. V. Kocharovsky, and V. V. Kocharovsky, Soviet Journal of Experimental and Theoretical Physics 107, 1049 (2008).

${ }^{32}$ L. P. J. Kamp, M. J. Kerkhof, F. W. Sluijter, and M. P. H. Weenink, Physics of Fluids B 4, 521 (1992).

${ }^{33}$ P. Braasch, Mathematical Methods in the Applied Sciences 20, 667 (1997).

${ }^{34}$ P. Braasch, Mathematical Methods in the Applied Sciences 21, 1015 (1998).

${ }^{35}$ P. Gratreau and P. Giupponi, Physics of Fluids 20, 487 (1977).

${ }^{36}$ B. C. Davidson and C. D. Striffler, Journal of Plasma Physics 12, 353 (1974).

${ }^{37}$ P. F. Ottinger and J. Guillory, Physics of Fluids 20, 1330 (1977).

${ }^{38}$ H. M. Lai, Physics of Fluids 23, 1559 (1980).

${ }^{39}$ S. Yoshikawa, Physical Review Letters 26, 295 (1971).

${ }^{40}$ P. L. Auer, Physics of Fluids 17, 148 (1974).

${ }^{41}$ J. R. Kan and H.-M. Lai, Physics of Fluids 15, 2041 (1972).

${ }^{42}$ J. P. Holdren, Physics of Fluids 12, 1059 (1969). 